\title{
Identifying the mechanisms of $\alpha$-synuclein-mediated cytotoxicity in Parkinson's disease: new insights from a bioinformatics-based approach
}

\author{
Sankha S Chakrabarti1 ${ }^{\text {iD }, ~ V e n k a t a d r i ~ S ~ S u n d e r ², ~ U p i n d e r ~ K a u r ~}{ }^{3}$, Sapna Bala1, Priyanka \\ Sharma $^{4}$, Manjari Kiran ${ }^{2}$, Ravindra K Rawal ${ }^{5}$ \& Sasanka Chakrabarti*,4 \\ ${ }^{1}$ Department of Geriatric Medicine, Institute of Medical Sciences, Banaras Hindu University, Varanasi, UP, India \\ ${ }^{2}$ Department of Systems \& Computational Biology, School of Life Sciences, University of Hyderabad, Telangana, India \\ ${ }^{3}$ Department of Pharmacology, All India Institute of Medical Sciences, Gorakhpur, UP, India \\ ${ }^{4}$ Department of Biochemistry \& Central Research Cell, Maharishi Markandeshwar (Deemed to be) University, Mullana, Ambala, \\ Haryana, India \\ ${ }^{5}$ Department of Chemistry, Maharishi Markandeshwar (Deemed to be) University, Mullana, Ambala, Haryana, India \\ *Author for correspondence: Tel.. +919874489805; profschakrabarti95@gmail.com
}

\begin{abstract}
Aim: A large body of evidence has implicated the cytotoxicity of $\alpha$-synuclein in Parkinson's disease (PD). We planned to use a bioinformatics-based approach to gain further insight into this process. Materials \& methods: Using STRING version 10, we identified interacting proteins of $\alpha$-synuclein. Using $\alpha$-synuclein and one of these interactors involved in apoptosis as query proteins, we identified other linked proteins. We further analyzed the interactions between some of these proteins by Protein-Protein Docking using ClusPro. Results: We identified BAX as an interacting protein of $\alpha$-synuclein. Interactions of $\alpha$-synuclein and BAX as well as BAX and BCL2L1 were determined. Conclusion: The interaction of $\alpha$-synuclein and BAX could play a crucial role in the cell death process of PD where apoptosis and mitochondrial permeability transition-driven necrosis may coexist.
\end{abstract}

First draft submitted: 19 March 2020; Accepted for publication: 29 April 2020; Published online: 10 July 2020

Keywords: cell death $\bullet$ docking $\bullet$ Parkinson's disease $\bullet$ protein-protein interaction $\bullet \alpha$-synuclein

Neurodegenerative diseases, such as Parkinson's disease (PD), Alzheimer's disease, amyotrophic lateral sclerosis etc., are characterized by the abnormal accumulation of different proteins, which are held responsible for an array of toxic actions leading to neuronal death [1,2]. In PD, abnormal accumulation of $\alpha$-synuclein (coded by the $S N C A$ gene) has been considered as a driving force of the disease pathogenesis, based on extensive findings from both animal and human studies [3,4]. $\alpha$-synuclein is a small acidic protein with a natively unfolded structure having $\mathrm{N}$-terminal lipid-binding segment with a tendency to form $\alpha$-helix on membrane binding, a central hydrophobic segment called non-amyloid $\beta$ component domain and a C-terminal acidic tail [4]. In the brain, it is located mainly at presynaptic terminals and is possibly involved in vesicular transport, neurotransmitter release and synaptic plasticity [4]. There has been some experimental evidence of localization of the protein in the neuronal nucleus and the mitochondria-associated endoplasmic reticulum membrane [5]. The role of accumulated monomeric or oligomeric forms of $\alpha$-synuclein (wild or mutant type) in causing mitochondrial dysfunction, endoplasmic reticulum stress, altered ER-Golgi transport, impaired vesicular transport, altered cytoskeletal dynamics, calcium dysregulation and oxidative stress have been suggested in multiple studies, but the molecular mechanisms of $\alpha$ synuclein action remains uncertain [3,6-11]. One suggested mechanism is the interaction of $\alpha$-synuclein with lipid biomembranes and disruption of membrane structure and integrity by oligomeric $\alpha$-synuclein [12]. On the other hand, $\alpha$-synuclein could interact with important proteins regulating cell structure, metabolism, differentiation and death $[7,10,13-15]$. The unequivocal identification of such interacting protein partners of $\alpha$-synuclein through isolation and characterization of protein complexes in different experimental models or in the actual disease 
condition has remained a formidable challenge and thus, a unique pathway of cell death mediated by $\alpha$-synuclein in PD is yet to be established. Added to this complexity is the uncertainty of the nature of neuronal death in PD. Although apoptosis has been advocated as the mechanism of cell death in PD, there is substantial evidence of involvement of other pathways of regulated cell death, including necrosis $[16,17]$. There has been interest in identifying the mechanism and nature of $\alpha$-synuclein-mediated cell death in model systems that could be relevant to PD pathogenesis $[3,7,18,19]$. One particularly interesting aspect in this context is the probable role played by $\alpha$-synuclein and mitochondria in cell death pathways like apoptosis, ferroptosis or regulated necrosis. In addition to the conventional experimental approach, we thought it interesting to use the vast bioinformatic resources available to predict a sequence of interactions involving $\alpha$-synuclein that may result in cell death, and then more effectively plan experimental designs to optimize resources and time.

The availability of high-throughput data collection techniques has resulted in the development of many databases and tools with varying degrees of complexity in which protein interactions are annotated at various levels of details. These can be utilized to understand protein-protein interaction (PPI) within the cells in normal and abnormal conditions $[20,21]$. Many complex cellular mechanisms, including programmed cell death pathways, take place through multiple and sequential interactions among different proteins. Such interactions generally lead to conformational changes in the interacting proteins causing activation of enzymatic activity (e.g., protein kinase) or translocation of the proteins to new compartments (e.g., from cytosol to mitochondria or nucleus) [22,23]. Such PPI networks may be generated with $\alpha$-synuclein as the query protein in STRING database to identify various interacting partners of $\alpha$-synuclein with links to cell death pathways. The STRING database, which is available online, has been designed in a user friendly and comprehensive manner covering nearly 5090 organisms and 24.6 million proteins. It provides us interactions, exact and predicted, based on experimental data as well as bioinformatics-based studies like gene fusion, gene co-occurrence, co-expression and text mining [24]. This database also provides us with information about predicted secondary and tertiary structures of large numbers of interacting proteins and the nature of their interactions through colored nodes and edges. For our present purpose, the most relevant PPI networks would be those that focus on experimentally proven interactions of $\alpha$-synuclein with proteins of programmed cell death pathways. However, such PPI networks would at best be predictive. We, therefore, thought it prudent to perform protein-protein docking, which could demonstrate the actual probabilities of physical interactions of proteins of interest. Protein docking is the prediction of the 3D structure of a protein-protein complex from the coordinates of its component structures. Of the various available approaches, rigid body docking of two proteins is commonly performed in the first stage among large number of possible conformations and in subsequent stages a small subset of structures are refined and re-ranked using other energy functions $[25,26]$. Various algorithms have been optimized for this purpose [27]. Using both these approaches in the current study, we have attempted to gain new insight on interactions of $\alpha$-synuclein with other proteins that may culminate in neuronal death.

\section{Materials \& methods}

\section{Creation of PPI network using STRING version 10}

The STRING version 10 database was used online to produce PPI network using $\alpha$-synuclein as the query protein and selecting Homo sapiens as the species. The PPI network obtained in STRING, shown as nodes connected by edges, was successively modified and refined by changing the parameter setting such as the number of interactor proteins, active interaction sources and minimum required interaction score. The PPI network from STRING was based on various actual and predicted interactions among $\alpha$-synuclein and its interacting partners. These included actual experimental physical interaction as well as predicted interactions based on gene fusion, gene co-expression, gene co-occurrence, text-mining, etc. After getting the initial PPI networks of $\alpha$-synuclein, we finally selected a network based on actual physical interactions with five of the most significant interacting proteins in the first shell. The database provided us the important details of the interacting proteins and we selected one protein linked with a programmed cell death pathway. We used the STRING database again utilizing two query proteins (i.e., $\alpha$-synuclein and the newly identified interacting partner of $\alpha$-synuclein) to obtain a final PPI network consisting of these two query proteins and their five significant interacting partners in the first shell.

\section{Protein-protein docking}

In order to verify further the physical interactions among these proteins, we performed protein-protein docking using the software ClusPro. ClusPro is a widely used software for direct docking of two proteins [28]. ClusPro first performs rigid body docking by sampling billions of conformations and then clusters 1000 lowest-energy structures 


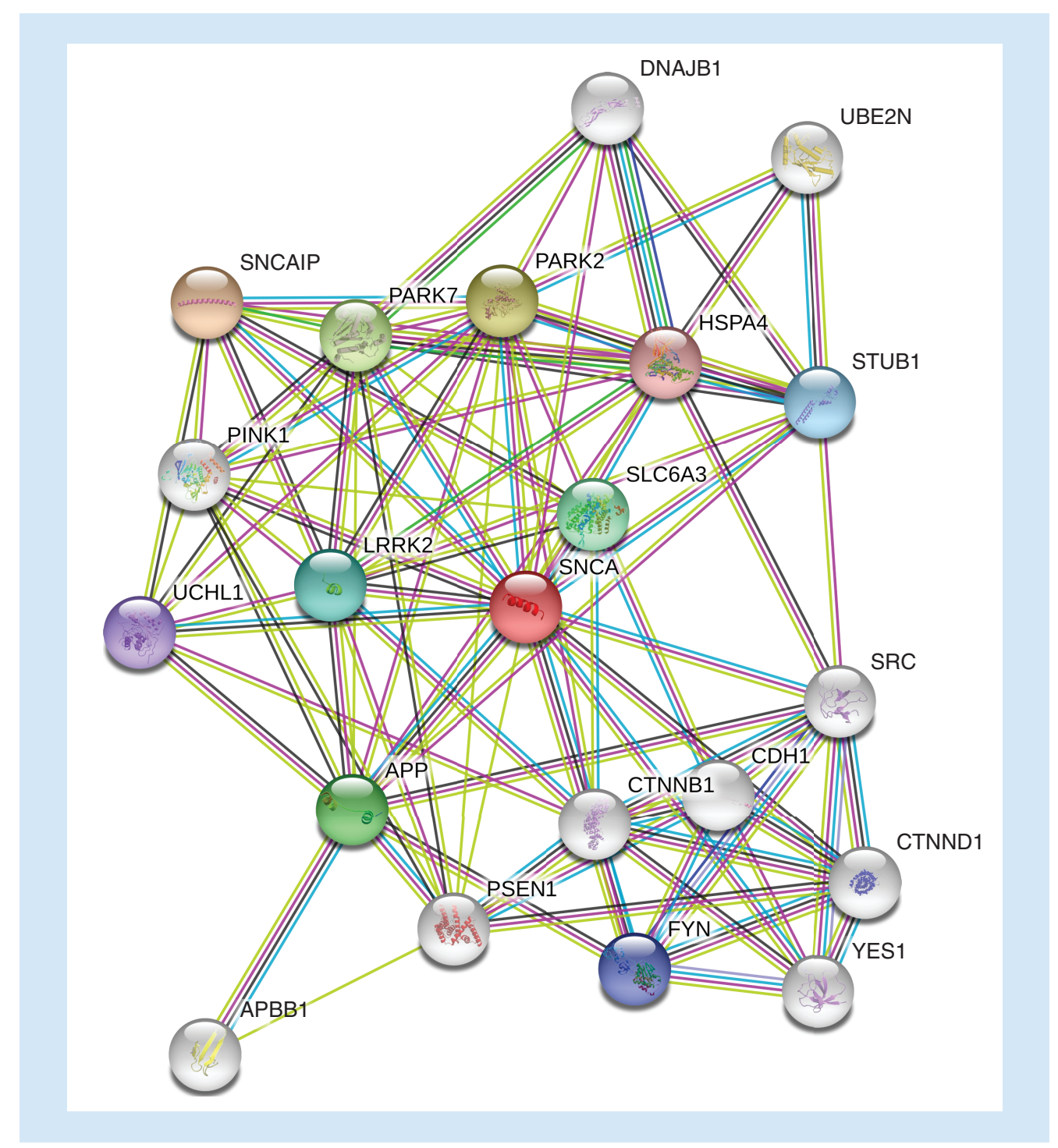

Figure 1. Expanded protein-protein interaction network of $\alpha$-synuclein obtained from STRING version 10. Nodes represent proteins and edges represent PPI. The meanings of node color and node content and color and thickness of edges are detailed in the database (https://string-db.org). Parameter setting: 20 interacting proteins (ten in the first shell, ten in the second shell); interaction score 0.40 (medium confidence); all active interaction sources as mentioned in the database.

PPI: Protein-protein interaction.

based on root-mean-square deviation. It also refines the selected structures by minimizing the CHARMM energy. The top ten docking structures were selected based on lowest energy, and the protein interactions calculator was used to identify different types of interactions including hydrophobic (interaction within $5 \mathrm{~A}$ ), hydrogen bonds, cation-pi, ionic and aromatic-aromatic interactions in all the ten models [29]. For the current study, the PDB structures PDB: 1XQ8, 1F16 and 1R2D for $\alpha$-synuclein, BAX and BCL2L1, respectively, were downloaded and used for docking.

\section{Results}

When the STRING version 10 database was searched using the query protein $\alpha$-synuclein selecting Homo sapiens as the species, a complex PPI network with 20 interacting proteins (ten in first shell and ten in second shell) could be seen (Figure 1). The nodes indicated the different interacting partners and the edges represented the nature of interaction. After changing the parameter setting of PPIs to include only co-expression and experimental physical 


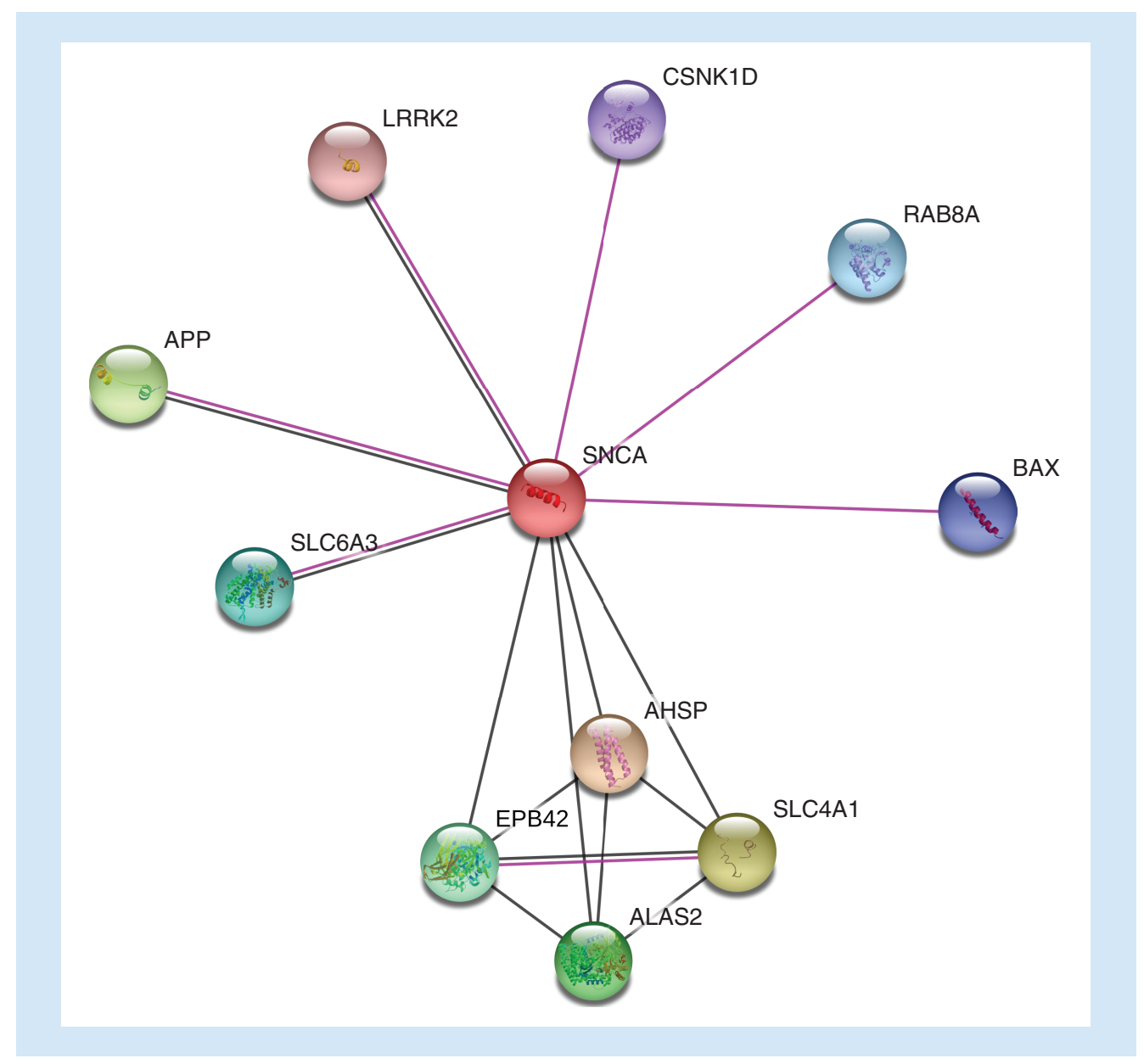

Figure 2. Refined protein-protein interaction network of $\alpha$-synuclein. Ten interacting proteins are seen; interaction score $(0.40)$ and active interaction source includes experimental physical interaction and gene co-expression.

interaction, the parameters most expected to be involved in actual pathophysiologic process, we obtained a PPI network shown in Figure 2. In this PPI network, there is experimental physical interaction between $\alpha$-synuclein and BAX which is a proapoptotic protein. In Figure 2, an important PD-related protein, LRRK2, was seen to interact with $\alpha$-synuclein through actual physical interaction and co-expression. We further refined the search by selecting most significant five interactor proteins having only experimental physical interactions (Figure 3). We checked the functions of the five interacting proteins present in Figure 3 from the STRING database, and the most important protein among these which was related to cell death was BAX. When the STRING database was searched with the two proteins BAX and $\alpha$-synuclein simultaneously with number of interactors set at five, we obtained the PPI network shown in Figure 4. In Figure 4, we observed that two important proteins of programmed cell death pathways, BCL2L11 and BCL2L1, physically interact with BAX with high interaction scores of 0.828 and 0.97 , respectively. On the other hand, BAX has direct physical interactions with $\alpha$-synuclein with moderate interaction score of 0.52 .

We further analyzed the protein-protein docking results between $\alpha$-synuclein and BAX, as well as between BAX and BCL2L1. The models obtained by docking showed that the 5th and 6th helices of BAX (PDB: 1F16) within BCL2 homology domain (Pfam: 63-158) were involved in interaction with $\alpha$-synuclein (PDB: 1XQ8). The interacting residues are 142Asp, 143Phe, 147Arg, 151Trp and 154Asp of BAX (Figure 5). Phe at position 4 in the first helix (3-11) of $\alpha$-synuclein also seemed to be important for the interaction with BAX. Amino acids 71-97 of five $\beta$-strands in the $\mathrm{C}$-terminal of $\alpha$-synuclein protein appeared to be another stretch that seemed to be interacting with BAX. The protein-protein docking between BAX (PDB: 1F16) and BCL2L1 (PDB: 1R2D) again showed 


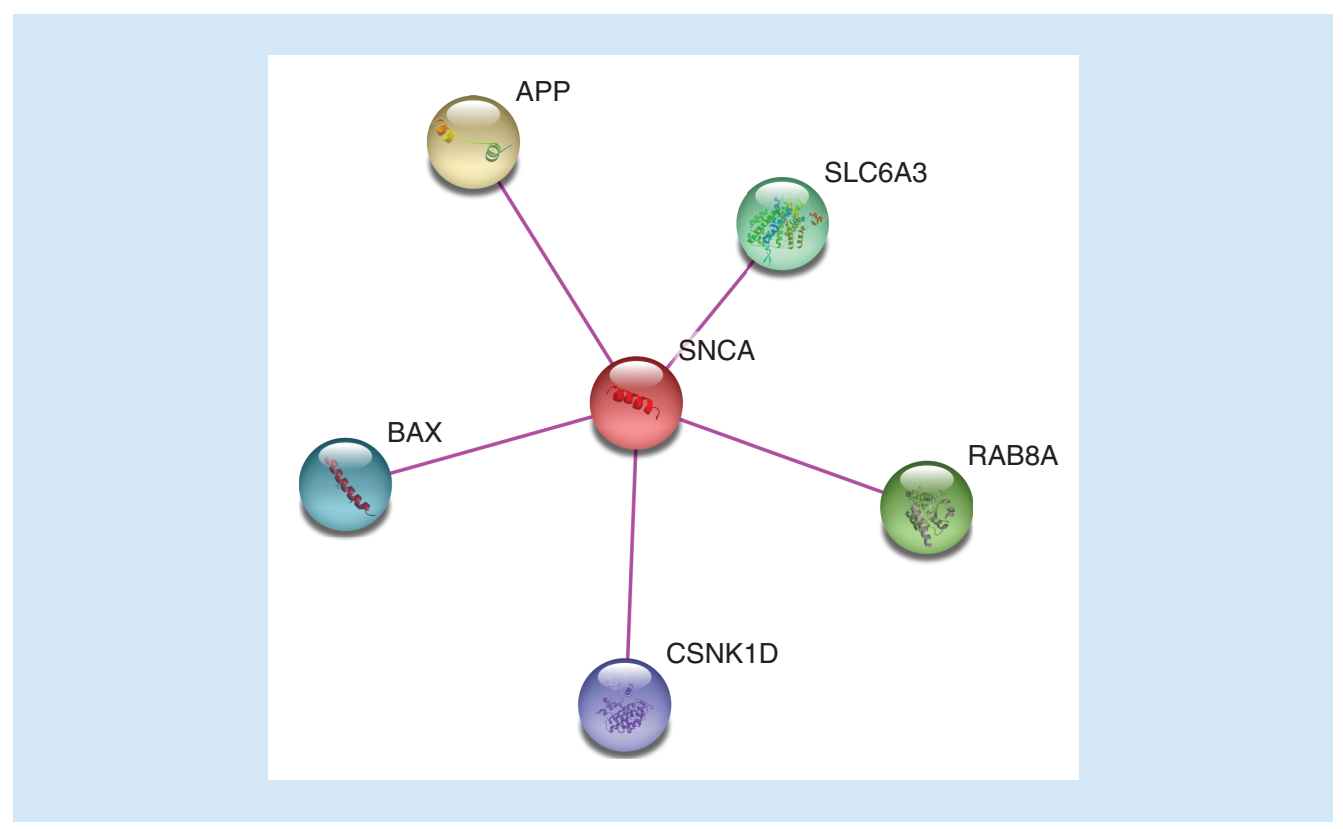

Figure 3. $\alpha$-Synuclein and limited interacting proteins obtained from STRING version 10. Five interacting proteins in protein-protein interaction network; interaction score set at 0.40 and active interaction source includes only experimental physical interaction.

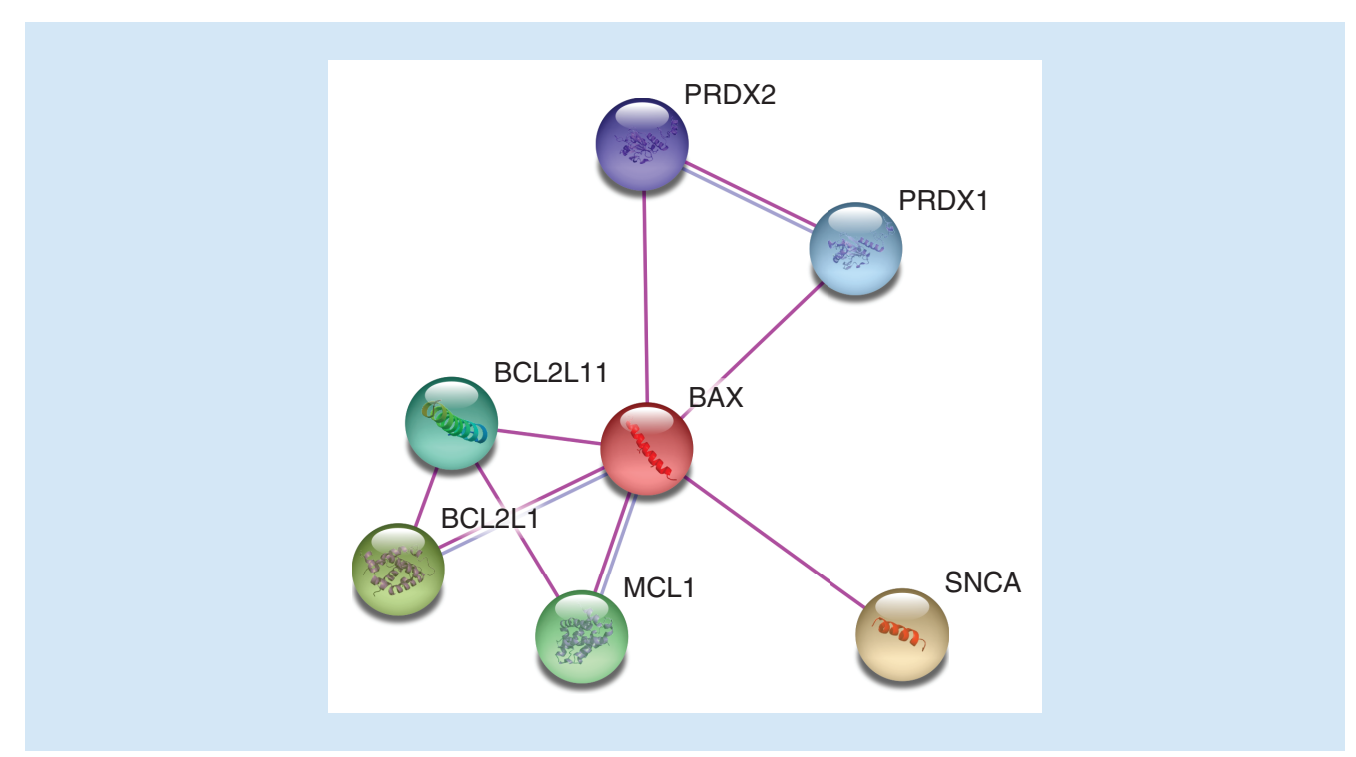

Figure 4. STRING database search results with two query proteins: $\alpha$-synuclein and BAX. Five interacting proteins of $\alpha$-synuclein and BAX can be seen; interaction score set at 0.40 (medium confidence) and active interaction source is only experimental physical interaction. Apart from BAX, two more proteins of BCL2 family BCL2L1 and BCL2L11 are also seen.

BCL-2 domain of BAX involved in interaction with BCL2L1. Furthermore, in BCL2L1 the residues Asp132 and Asp133 were found to be involved in interaction in more than $50 \%$ of the top ten docking models.

\section{Discussion}

The understanding of pathogenesis, identification of risk factors and development of biomarkers and neuroprotective drugs for PD remains a challenging job [3,30]. In particular, there are many areas of uncertainties related to the cause, mechanisms and nature of neuronal death in PD. However, the involvement of $\alpha$-synuclein and 


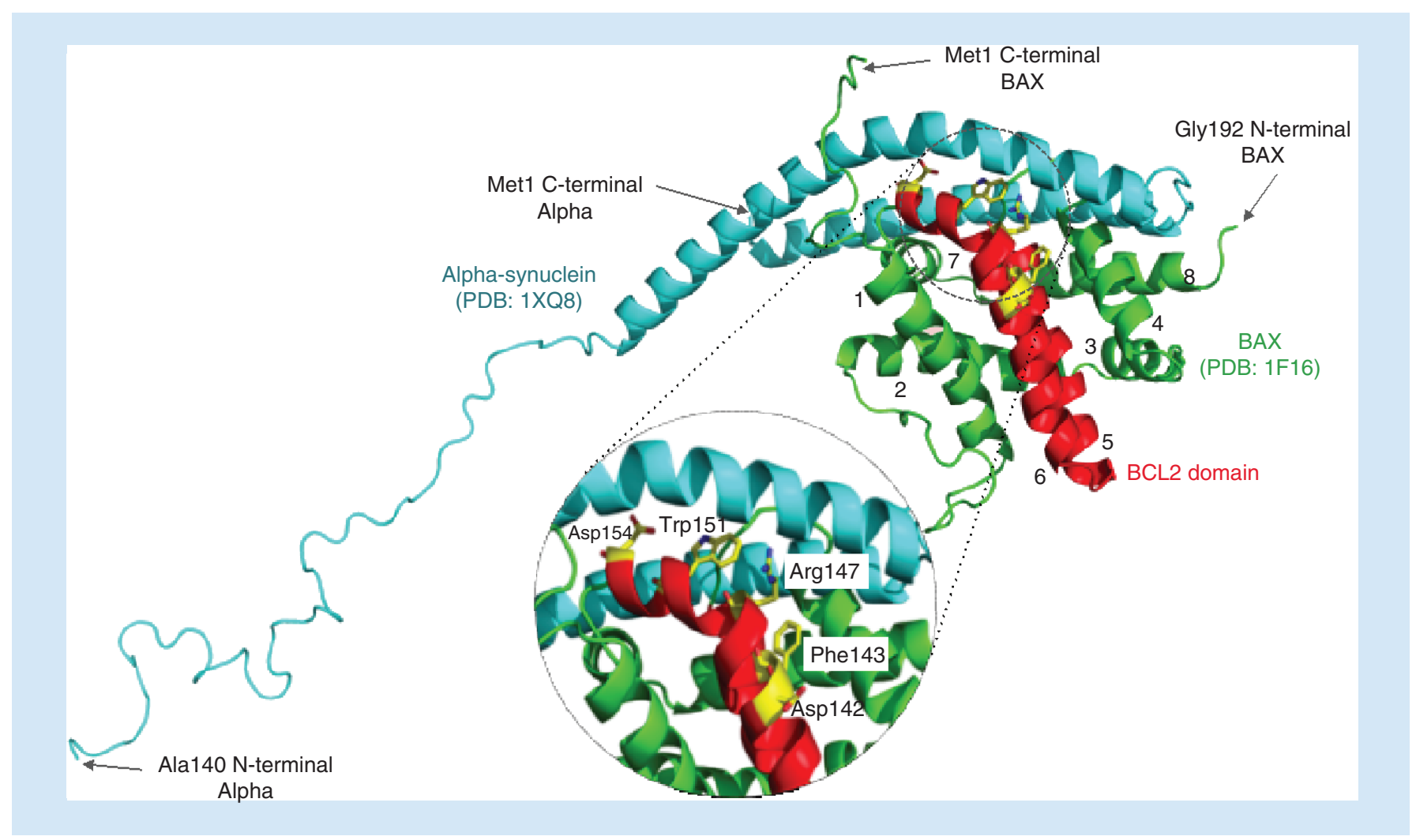

Figure 5. Docking study of $\alpha$-synuclein (PDB: 1XQ8) and Bax (PDB: 1F16) using ClusPro. ClusPro best model obtained after docking is shown as ribbon diagram using PyMol (an open source molecular visualization system). $\alpha$-synuclein is shown in cyan color and BAX in green color, whereas BCL2 homology domain is highlighted in red. The numbers represent the helices (1-8) in BAX starting from the $\mathrm{N}$-terminus. The residues involved in interaction are shown with line and stick model Asp142, Phe143, Arg147, Trp151, Asp154 of BAX.

mitochondrial dysfunction in this process has been widely documented. We have been exploring this aspect in a model of neural cell death in which the intracellular level of $\alpha$-synuclein is raised by different nongenetic manipulations $[7,18,19]$. In the current study, we have attempted to find out how a bioinformatics-based search could assist us in identifying the mechanisms and nature of cell death in PD neurodegeneration. Our results first showed that $\alpha$-synuclein has an interacting partner, BAX, which is a proapoptotic protein belonging to the BCL2 family [31]. This interaction of $\alpha$-synuclein and BAX was further validated by protein-protein docking studies, which showed that $\mathrm{BH} 3$ domain of $\mathrm{BAX}$ is involved in the interaction process. BAX is a cytosolic protein which upon activation translocates to the mitochondria, undergoes oligomerization and induces apoptosis by permeabilization of mitochondrial outer membrane and releasing proteins, such as cytochrome c, Smac/DIABLO, AIF, etc., leading to apoptosis [31]. The trigger for the activation of BAX and subsequent translocation to mitochondria is unknown but a conformational change of BAX is possibly required [31,32]. Our results tend to suggest that the binding of $\alpha$-synuclein to BAX with a presumable conformational change in BAX may be the initiating event. Furthermore, $\alpha$-synuclein, though a cytosolic protein, is capable of entering mitochondria and thus it is plausible that a complex of $\alpha$-synuclein-BAX would reach the mitochondria as the initiating event of cell death. It is interesting that the interaction of $\alpha$-synuclein with BAX takes place at the BCL2 homology domain, which is generally considered to be the region where $\mathrm{BH} 3$-only protein can bind and activate BAX $[32,33]$. The interaction of BAX with BCL2L1 that is an antiapoptotic protein is also validated by protein-protein docking in our current study. This interaction in normal conditions might lead to inactivation of pro-apoptotic function of BAX. However, it is plausible that, under various stressful conditions $\alpha$-synuclein binds with BAX and prevents BAX-BCL2L1 interactions, leading to uninhibited pro-apoptotic action of BAX.

Another interesting area of controversy for PD neurodegeneration is related to the nature of cell death. Apoptosis has been widely recognized as the pathway of neuronal death in PD, but the evidence for other modes of cell death in PD is also substantial [16,17]. It has been shown that, under a variety of conditions, the death of SH-SY5Y 
cells is mediated by $\alpha$-synuclein-induced mitochondrial permeability transition (MPT) pore activation, which can be prevented by cyclosporine $[7,18,19]$. This mode of cell death is recognized as MPT-necrosis which could be, in our opinion, a potential mechanism of PD neurodegeneration [34]. It appears that different cell death mechanisms may function in concert in the degenerating dopaminergic neurons in the PD brain. Extending our hypothesis of translocation of $\alpha$-synuclein-BAX complex to the mitochondria, it is thus tempting to speculate that $\alpha$-synuclein initiates an apoptotic type of cell death indirectly through BAX, which causes mitochondrial outer membrane permeabilization, and directly through a MPT-necrosis process.

\section{Conclusion}

Our bioinformatics-based study has provided new clues to understand the neurodegeneration of PD in model systems. However, these possibilities of neuronal damage envisaged from bioinformatics-based analysis involving $\alpha$-synuclein, BCL family of proteins and mitochondria are only predictive in nature, and experimental validation and biochemical characterizations of PPIs are necessary in suitable models to establish their role in actual PD pathogenesis.

\section{Future perspective}

Utilizing bioinformatics-based approaches to understand the pathogenesis of diseases, especially neurodegenerative diseases, is an emerging area with great potential. The current study has indicated how information gained from database searching and protein-protein docking studies can be utilized to formulate a pathway of neuronal death in PD. This will help us to design suitable experiments, optimizing resources and time, to obtain definitive information on the molecular pathogenesis of PD. Without this information, experimental studies usually progress slowly by trial and error approach. Thus, bioinformatics-based methods are an attractive alternative that may be utilized for other neurological diseases as well. We anticipate more such similar studies in the future, but many refinements and critical analyses will be required to develop this system fully.

\section{Summary points}

- This bioinformatics-based study aimed to identify a pathway of neuronal damage initiated by $\alpha$-synuclein, which could be relevant to Parkinson's disease (PD) pathogenesis.

- Protein-protein interaction (PPI) networks were constructed on STRING version 10 using $\alpha$-synuclein as the query protein.

- The PPI networks were refined successively by changing parameter settings and focusing on experimental evidence and co-expression data. A final network of $\alpha$-synuclein and five interacting proteins, including BAX, $B C L 2 L 1$ and BCL2L11, involved in the apoptotic pathway was obtained.

- Protein-protein docking studies were carried out between $\alpha$-synuclein and BAX as well as between BAX and BCL2L1 to suggest possible molecular interactions among these proteins.

- Analyzing the data, we suggest that $\alpha$-synuclein and BAX interactions followed by translocation of the complex to mitochondria may trigger apoptotic neuronal death. This may have implications in the neurodegeneration of PD.

- These predictive data need experimental validation in suitable models.

Financial \& competing interests disclosure

SS Chakrabarti wishes to thank the Indian Council of Medical Research for research funding; S Chakrabarti wishes to thank the Maharishi Markandeshwar (deemed to be) University, Mullana, Haryana and the Indian Council of Medical Research for research funding. The authors have no other relevant affiliations or financial involvement with any organization or entity with a financial interest in or financial conflict with the subject matter or materials discussed in the manuscript apart from those disclosed.

No writing assistance was utilized in the production of this manuscript.

\section{Open access}

This work is licensed under the Attribution-NonCommercial-NoDerivatives 4.0 Unported License. To view a copy of this license, visit http://creativecommons.org/licenses/by-nc-nd/4.0/

\section{References}

Papers of special note have been highlighted as: $\bullet$ of interest

1. Soto C. Unfolding the role of protein misfolding in neurodegenerative diseases. Nat. Rev. Neurosci. 4(1), 49-60 (2003).

2. Chung CG, Lee H, Lee SB. Mechanisms of protein toxicity in neurodegenerative diseases. Cell. Mol. Life Sci. 75(17), 3159-3180 (2018). 
3. Ganguly U, Chakrabarti SS, Kaur U, Mukherjee A, Chakrabarti S. Alpha-synuclein, proteotoxicity and Parkinson's disease: search for neuroprotective therapy. Curr. Neuropharmacol. 16(7), 1086-1097 (2018).

4. Stefanis L. $\alpha$-Synuclein in Parkinson's disease. Cold Spring Harb. Perspect. Med. 2(2), a009399 (2012).

5. Surguchov A. Intracellular dynamics of synucleins: "here, there and everywhere". Int. Rev. Cell Mol. Biol. 320, 103-169 (2015).

6. Ghiglieri V, Calabrese V, Calabresi P. Alpha- synuclein: from early synaptic dysfunction to neurodegeneration. Front. Neurol. 9,295 (2018).

- A good up-to-date account of role of $\alpha$-synuclein in Parkinson's disease (PD) pathogenesis.

7. Ganguly U, Ganguly A, Sen O et al. Dopamine cytotoxicity on SH-SY5Y cells: involvement of $\alpha$-synuclein and relevance in the neurodegeneration of sporadic Parkinson's disease. Neurotox. Res. 35(4), 898-907 (2019).

8. Zhang G, Xia Y, Wan F et al. New perspectives on roles of alpha-synuclein in Parkinson's disease. Front. Aging Neurosci. 10, 370 (2018).

9. Smith WW, Jiang H, Pei Z et al. Endoplasmic reticulum stress and mitochondrial cell death pathways mediate A53T mutant alpha-synuclein-induced toxicity. Human Mol. Genet. 14(24), 3801-3811 (2005).

10. Ordonez DG, Lee MK, Feany MB. $\alpha$-Synuclein induces mitochondrial dysfunction through spectrin and the actin cytoskeleton. Neuron 97(1), 108-124 (2018).

11. Gallegos S, Pacheco C, Peters C, Opazo CM, Aguayo LG. Features of alpha-synuclein that could explain the progression and irreversibility of Parkinson's disease. Front. Neurosci. 9, 59 (2015).

12. Pfefferkorn CM, Jiang Z, Jennifer C, Lee JC. Biophysics of $\alpha$-synuclein membrane interactions. Biochim. Biophys. Acta 1818(2), 162-171 (2012).

13. Emamzadeh FN. Alpha-synuclein structure, functions, and interactions. J. Res. Med. Sci. 21, 29 (2016).

14. Schnack C, Danzer KM, Gillardon BHF. Protein array analysis of oligomerization-induced changes in alpha-synuclein protein-protein interactions points to an interference with Cdc42 effector proteins. Neuroscience 154(4), 1450-1457 (2008).

15. Jellinger KA. Interaction between $\alpha$-synuclein and other proteins in neurodegenerative disorders. Sci. World J. 11, 1893-1907 (2011).

16. Venderova K, Park DS. Programmed cell death in Parkinson’s disease. Cold Spring Harb. Perspect. Med. 2(8), a009365 (2012).

17. Perier C, Bové J, Vila M. Mitochondria and programmed cell death in Parkinson's disease: apoptosis and beyond. Antioxid. Redox Signal. 16(9), 883-895 (2012).

18. Bir A, Sen O, Anand S et al. $\alpha$-Synuclein induced mitochondrial dysfunction in isolated preparation and intact cells: implications in the pathogenesis of Parkinson's disease. J. Neurochem. 131(6), 868-877 (2014).

19. Ganguly U, Banerjee A, Chakrabarti SS et al. Interaction of $\alpha$-synuclein and Parkin in iron toxicity on SH-SY5Y cells: implications in the pathogenesis of Parkinson's disease. Biochem. J. 477(6), 1109-1122 (2020).

- It links several pathogenic elements of PD neurodegeneration like iron accumulation, oxidative stress, $\alpha$-synucleinopathy and mitochondrial dysfunction in a common pathway.

20. Droit A, Poirier GG, Hunter JM. Experimental and bioinformatic approaches for interrogating protein-protein interactions to determine protein function. J. Mol. Endocrinol. 34(2), 263-280 (2005).

21. Kuzmanov U, Emili A. Protein-protein interaction networks: probing disease mechanisms using model systems. Genome Med. 5(4), 37 (2013).

- It gives a sound description of various experimental approaches and models to study protein-protein interactions. It also describes the principles of bioinformatics-based approaches in simple terms in predicting protein-protein interactions.

22. Pawson T, Nash P. Protein-protein interactions define specificity in signal transduction. Genes Dev. 14(9), 1027-1047 (2000).

23. Kolch W. Meaningful relationships: the regulation of the Ras/Raf/MEK/ERK pathway by protein interactions. Biochem. J. 351(Pt 2), 289-305 (2000).

24. Szklarczyk D, Franceschini A, Wyder S et al. STRING v10: protein-protein interaction networks, integrated over the tree of life. Nucleic Acids Res. 43(Database Issue), D447-D452 (2015).

25. Camacho CJ, Gatchell DW, Kimura SR, Vajda S. Scoring docked conformations generated by rigid-body protein-protein docking. Proteins 40(3), 525-537 (2000).

26. Weng Z, Vajda S, Delisi C. Prediction of protein complexes using empirical free energy functions. Protein Sci. 5(4), $614-626$ (1996).

27. Halperin I, Ma B, Wolfson H, Nussinov R. Principles of docking: an overview of search algorithms and a guide to scoring functions. Proteins 47(4), 409-443 (2002).

28. Kozakov D, Hall DR, Xia B et al. The ClusPro web server for protein-protein docking. Nat. Protocols. 12(2), 255-278 (2017).

- A very good paper to understand how ClusPro works for Protein-Protein Docking studies.

29. Tina KG, Bhadra R, Srinivasan N. PIC: protein interactions calculator. Nucleic Acids Res. 35(Web server issue), W473-W476 (2007).

30. Emamzadeh FN, Surguchov A. Parkinson's disease: biomarkers, treatment, and risk factors. Front. Neurosci. 12, 612 (2018).

- It is particularly good in dealing with PD risk factors and imaging studies in PD patients. 
31. Dewson G, Kluck RM. Mechanisms by which Bak and Bax permeabilise mitochondria during apoptosis. J. Cell Sci. 122(pt 16), 2801-2808 (2009).

32. Lalier L, Cartron P-F, Olivier C et al. Prostaglandins antagonistically control Bax activation during apoptosis. Cell Death Differen. 18(3), 528-537 (2011).

33. Bouillet $\mathrm{P}$, Strasser A. BH3-only proteins - evolutionarily conserved proapoptotic Bcl-2 family members essential for initiating programmed cell death. J. Cell Sci. 115(Pt 8), 1567-1574 (2002).

34. Choi ME, Price DR, Ryter SW, Choi AMK. Necroptosis: a crucial pathogenic mediator of human disease. JCI Insight 4(15), e128834 (2019). 